\title{
Slowing-Down and Stopped Charged Particles Cause Angular Dependence for Absorbed Dose Measurements
}

\author{
Amir A. Bahadori ${ }^{\mathrm{a}, *}$, Rajarshi Pal Chowdhury ${ }^{\mathrm{a}}$, Martin Kroupa ${ }^{\mathrm{b}}$, \\ Thomas Campbell-Ricketts ${ }^{\mathrm{c}}$, Ana Firan ${ }^{\mathrm{b}}$, Dan J. Fry ${ }^{\mathrm{d}}$, Ramona Gaza ${ }^{\mathrm{b}}$, \\ Stuart P. George ${ }^{\mathrm{c}}$, Lawrence S. Pinsky ${ }^{\mathrm{c}}$, Nicholas N. Stoffle ${ }^{\mathrm{b}}$, Ryan R. Rios ${ }^{\mathrm{b}}$, \\ Cary J. Zeitlin ${ }^{\mathrm{b}}$ \\ ${ }^{a}$ Department of Mechanical and Nuclear Engineering, Kansas State University, Manhattan, \\ KS 66506, USA \\ ${ }^{b}$ Leidos Innovations Corporation, Houston, TX 77058, USA \\ ${ }^{c}$ Department of Physics, University of Houston, Houston, TX 77004, USA \\ ${ }^{d}$ Space Radiation Analysis Group, NASA Johnson Space Center, Houston, TX 77058, USA
}

\begin{abstract}
The space radiation environment is dominated by heavy charged particles with atomic numbers ranging from 1 to 93 , with broad energy spectra that exceed $10 \mathrm{GeV}$ per nucleon. Despite advances in space radiation modeling and transport, radiation detectors continue to provide critical data for understanding risks of health effects to astronauts in space. In the past, NASA relied on tissueequivalent proportional counters and passive devices for operational dosimetry; however, in recent years, pixel detectors providing detailed information about the radiation environment through analysis of charged particle tracks have been demonstrated in space. These next-generation detectors, based on Timepix read-out technology, require special analysis considerations that were not necessary or possible for previous dosimetry tools. The impacts of slowing-down and stopped ions on absorbed dose measurements must be explicitly modeled to understand variations with detector orientation. The purpose of the present study is to conclusively demonstrate that while absorbed dose measurements of penetrating charged particles are independent of detector orientation, slowing-down and stopped particles can result in charged particle absorbed dose measure-
\end{abstract}

\footnotetext{
* Corresponding author

Email address: bahadori@ksu.edu (Amir A. Bahadori)
}

Preprint submitted to Radiation Physics and Chemistry

April 24, 2018 
ments that are dependent on detector orientation. Monte Carlo simulations of an unshielded detector, irradiated at selected orientations by different kinetic energy domains with fluence spectra representative of two historical solar particle events, are presented to demonstrate the dependence of absorbed dose measurements. Next, results from Monte Carlo simulations of the same energy domains and fluence spectra, isotropically impinging on an anisotropic shield configuration about the detector, are shown, to exhibit the potential for observing varying absorbed doses under realistic environment and shielding conditions. Finally, slowing-down and stopped proton data acquired with Timepix-based detectors at the Tandem Van de Graaff at Brookhaven National Laboratory are used to demonstrate the effect via accelerator-based measurements.

Keywords: Space radiation, Timepix, Dosimetry, Solar particle event, Protons, Monte Carlo

PACS: 07.77.Ka, 24.10.Lx, 29.40.Gx, 29.40.Wk, 34.50.Bw, 87.53.Bn, 96.50.Vg

\section{Introduction}

\section{1.1. Space Radiation Environment and Risks}

The space radiation environment is more intense than, and distinct from, the background radiation environment on Earth, primarily due to the absence of shielding provided by the combined effects of Earth's geomagnetic field and atmosphere. Three major sources of radiation in space are solar particle events (SPEs), galactic cosmic rays (GCRs), and geomagnetically-trapped particles. SPEs, which may last from hours to days, are comprised of charged ions, largely protons, accelerated through explosive processes on the Sun, such as solar flares and coronal mass ejections [1]. Event-integrated SPE fluence spectra are commonly modeled with proton kinetic energies up to about $2 \mathrm{GeV}$ for space radiation dosimetry purposes. GCRs comprise a slowly varying, low flux density background of fully-ionized heavy charged particles with broad energy spectra 2. Bands of geomagnetically-trapped protons and electrons form the Van Allen radiation belts 3 . The inner belt, part of which is present at Interna- 
tional Space Station (ISS) altitudes in the form of the South Atlantic Anomaly (SAA), is dominated by protons with kinetic energies less than about $1 \mathrm{GeV}$, while the outer belt is dominated by electrons with energies less than $20 \mathrm{MeV}$.

Scientists have been aware of human health risks arising from ionizing radiation exposure since the beginning of the 20th Century [4]. Astronauts on space missions are at risk of a number of radiation-induced health effects, including cancer [5], central nervous system damage [6], cardiovascular system effects [7, 8], cataracts [9, 10, and potentially Acute Radiation Syndrome (ARS) [11]. NASA's radiation protection program is similar to radiation protection programs on Earth in that it is based on the principles of justification, limitation, and minimization: exposures are justified in the context of individual and societal risks and benefits [12]; risk limits are imposed by NASA standards [13]; and the As Low As Reasonably Achievable (ALARA) Principle is applied prior to and during space missions [13, 14. Strategies for reducing lifetime impacts of radiation health effects include crew selection, shielding, development and use of radio-protectants, and environment prediction and monitoring. While space radiation environment models have significantly improved with time, environment monitoring remains an important tool for anchoring models to reality. Additionally, no method to accurately predict SPE occurrence and resulting proton flux density spectrum as a function of time presently exists, so active detection is particularly imperative for protecting astronauts venturing outside of Earth's geomagnetic field.

\subsection{Exploration Mission Radiation Monitoring}

The suite of NASA instruments used for space radiation protection on the ISS includes passive dosimeters [15]; tissue equivalent proportional counters (TEPCs) [15, 16]; ISS Radiation Assessment Detector (RAD), based on the Mars Science Laboratory RAD [17, with an additional sensor dedicated to neutron measurement; and Extra-Vehicular Charged Particle Directional Spectrometers (EV-CPDSs) [18]. These instruments require either mass transfer to and from Earth, in the case of passive dosimeters, or large mass, volume, and 
power budgets, in the case of active detectors. Space radiation monitoring for exploration missions, however, necessitates use of active detectors that are much lower mass and volume, and can operate on less power.

In 2012, NASA began a technology demonstration, known as the ISS Radiation Environment Monitor (REM), to investigate the feasibility of designing a space radiation monitoring solution based on the Timepix chip [19] developed through the Medipix2 Collaboration at CERN [20. The Timepix chip is a read-out array of 256-by-256 pixels, each with a side length of $55 \mu \mathrm{m}$. For space radiation dosimetry, it is bump-bonded to bulk silicon semiconductor, which provides an active volume for energy deposition by ionizing radiation. Pixel-wise calibration of the devices is traditionally performed using low-energy X-rays and gammas [21, although recently, a method to augment the traditional calibration with higher energy depositions relevant for space radiation has been devised [22].

The Timepix detection assembly (consisting of the Timepix read-out chip and silicon detection element) is an attractive option for space radiation environment monitoring because it is small, can be operated at less than one watt, and provides an energy deposition pattern for charged particles that traverse the active volume. This energy deposition pattern can be spatially integrated to generate traditional data products, such as absorbed dose, or can be processed using more complicated analysis tools to probabilistically determine the charge and kinetic energy of the incident ion. Generally, ISS REM units have shown good agreement with the ISS-TEPC 23, 24]. They have also shown good agreement with newer space radiation detectors, such as DOSTEL, ISS RAD, and intra-vehicular (IV) TEPC. Given the success of the ISS REM technology demonstration, NASA continued pursuing Timepix-based radiation monitoring solutions, including the Battery-operated Independent Radiation Detector (BIRD), which flew on Exploration Flight Test 1 (EFT-1) in December 2014 [25], and the Hybrid Electronic Radiation Assessor (HERA), which will fly on Exploration Mission 1 (EM-1) and EM-2, the first crewed mission of the Orion Multi-Purpose Crew Vehicle. 


\subsection{Problem and Hypothesis}

Attempts to model ISS REM detector responses have resulted in underestimation of measured absorbed dose [26. Also, the absorbed dose reported by ISS-TEPC has been observed to vary as a function of angular position at the same location on ISS [27]. Furthermore, differences in absorbed dose have been observed between co-located DOSTEL active silicon dosimeters [28]. Previous absorbed dose differences have been attributed to ISS orientation changes in orbit 29], but no study has conclusively determined the portion of the proton spectrum responsible for the changes, nor has the potential for the effect been examined in isotropic space radiation environments.

Slowing-down and stopped ion ${ }^{1}$ have been previously shown to cause artifacts in $d E / d x$ spectra acquired by the ISS REM on-orbit [30. Additionally, impacts of detector orientation on neutron detector efficiency, resulting from non-uniform interaction probabilities for different path lengths, have been detailed in literature 31. We hypothesize that the change in $d E / d x$ over the path length causes an angular dependence for absorbed dose measurements for non-isotropic detector volumes, such as the silicon detection element used in NASA Timepix-based radiation detection systems. Thus, impacts of slowingdown and stopped ions must be considered by modeling the entire detector volume to properly simulate detector response or use these data for astronaut radiation risk analyses.

\section{Materials and Methods}

\subsection{Consideration of Penetrating Charged Particles}

Since slowing-down and stopped charged particles are hypothesized to cause angular dependence for absorbed dose, it is first useful to eliminate penetrating

\footnotetext{
${ }^{1}$ In the present study, "slowing-down" is used to describe ions with a substantial change in $d E / d x$ over a given path length, while "stopped" is used to describe ions that slow down to zero kinetic energy in the detector volume. "Penetrating" is used to describe ions that exhibit minimal change in $d E / d x$ over a given path length.
} 
charged particles as contributors to such an effect. To show that absorbed dose from penetrating charged particles is invariant with angular distribution, several assumptions are employed:

1. Energy deposited is equal to energy absorbed;

2. The field is broad beam;

3. Absorbed dose contributions from nuclear interactions are small relative to absorbed dose contributions resulting from Coulombic interactions with atomic electrons;

4. Straggling is neglected, so energy deposited per unit path length is equal to linear energy transfer (LET); and

5. The energy deposited is much less than the kinetic energy of the particle, so the energy deposited per unit path length does not change over the path.

Assumptions 4 and 5 are notably inapplicable to slowing-down and stopped charged particles. Assumption 3 is less true for slowing-down and stopped charged particles than for penetrating charged particles, but the maximum contribution of nuclear interactions to stopping power is about $10 \%$ 32.

Here, the active volume of the detector is modeled as a rectangular parallelepiped, which is subjected to irradiation from an arbitrary solid angle direction. The absorbed dose, $D$, for the active volume is given as

$$
D=\frac{1}{m} \sum_{Z} \int_{T} \int_{\boldsymbol{\Omega}} E(Z, T, \boldsymbol{\Omega}) d T d \boldsymbol{\Omega}
$$

where $m$ is the mass of the active volume, $Z$ represents the particle type, $T$ is the particle kinetic energy, $E$ is the energy deposition, and $\boldsymbol{\Omega}$ is the solid angle direction. We consider one type of particle and a mono-energetic beam, so the absorbed dose calculation simplifies to

$$
D=\frac{1}{m} \int_{\boldsymbol{\Omega}} E(\boldsymbol{\Omega}) d \boldsymbol{\Omega}
$$


Since the LET is constant for all path lengths traversing the active volume, the energy deposition is found as the product of LET, $S$, and the integral path length, $\int_{\ell} \ell N_{\boldsymbol{\Omega}}(\ell) d \ell$. So,

$$
D=\frac{1}{m} S \int_{\boldsymbol{\Omega}} \int_{\ell} \ell N_{\boldsymbol{\Omega}}(\ell) d \ell d \boldsymbol{\Omega}
$$

29 Using the definition of mean path length, $\bar{\ell}_{\boldsymbol{\Omega}}$,

$$
D=\frac{1}{m} S \int_{\boldsymbol{\Omega}} \bar{\ell}_{\boldsymbol{\Omega}} \int_{\ell} N_{\boldsymbol{\Omega}}(\ell) d \ell d \boldsymbol{\Omega}
$$

Now, the integral of the path length distribution must be equal to the number of particles incident on the active volume, $N_{\Omega}$. Additionally, the mean path length through the detector is equal to the quotient of the volume, $V$, and projected area, $A_{\Omega}$ [33]. Thus,

$$
D=\frac{1}{m} S V \int_{\boldsymbol{\Omega}} \frac{N_{\boldsymbol{\Omega}}}{A_{\boldsymbol{\Omega}}} d \boldsymbol{\Omega}
$$

The quotient of the number of particles incident on the active volume and the projected area is the particle fluence as a function of solid angle direction, $\Phi(\boldsymbol{\Omega})$. Now,

$$
D=\frac{1}{m} S V \int_{\boldsymbol{\Omega}} \Phi(\boldsymbol{\Omega}) d \boldsymbol{\Omega}
$$

Integrating over solid angle and using the definition of mass density, $\rho$,

$$
D=\frac{1}{\rho} S \Phi
$$

Equation 7 exhibits no solid angle direction dependence $2^{2}$ Therefore, absorbed dose from penetrating charged particles is invariant with solid angle distribution. This result is true for environments consisting of different particle types and energies, provided they satisfy the stated assumptions, since absorbed dose for

\footnotetext{
${ }^{2} \mathrm{An}$ identical result is found by defining particle fluence as the path length per unit volume in the detector. Additionally, Equation 7 is used to calculate absorbed dose to a point in the NASA High Charge and Energy Transport Code (HZETRN) 34, 35.
} 
the environment can be found by integrating over the distribution of particle types and energies.

\subsection{Radiation Transport}

Since fast, penetrating particles satisfying the five assumptions listed in the previous section can not be the source of angular dependence in absorbed dose, it is reasonable to investigate the impacts of slowing-down and stopped charged particles, especially since Assumption 5 is, by definition, inapplicable to slowing-down and stopped ions. To examine the differences in absorbed dose from slowing-down and stopped ions with solid angle distribution, the Particle and Heavy Ion Transport code System (PHITS) 36 38 version 2.88 was used. PHITS is deployed on the Kansas State University Beocat High Performance Computing System using the Message Passing Interface to permit fast execution over multiple computing cores.

Heavy charged particles were transported to $1 \mathrm{keV}$ per nucleon, photons were transported to $1 \mathrm{keV}$, and electrons and positrons were transported to $100 \mathrm{keV}$. The PHITS event generator was used to statistically generate and transport reaction products resulting from neutrons with kinetic energies below $20 \mathrm{MeV}$. Delta rays were not transported, as delta ray escape is not expected to be a substantial contributor to lack of charged particle equilibrium for the space radiation environments considered. Energy and angle straggling were considered in all simulations.

\subsection{Shielding and Irradiation Conditions}

Three scenarios were established to investigate the role of slowing-down and stopped charged particles in angular dependence of absorbed dose. These are:

1. An unshielded detector irradiated by two historical SPEs with monodirectional angular distributions,

2. A detector enveloped by an anisotropic shield distribution irradiated by two historical SPEs (same as those used in Case 1) with isotropic angular distributions, and 
3. An unshielded detector irradiated by a broad, mono-directional beam of mono-energetic protons.

Case 1 is an extreme scenario that is expected to yield the largest differences in absorbed dose as a function of incident angle. Three angles with respect to normal incidence on the detector face were considered: $0^{\circ}, 45^{\circ}$, and $90^{\circ}$. The polar angle was constant at $0^{\circ}$. Case 2 represents a more realistic scenario, where the detector is shielded by an aluminum cylindrical shell with wall thickness $1 \mathrm{~g} \mathrm{~cm}^{-2}$, end thickness $20 \mathrm{~g} \mathrm{~cm}^{-2}$, inner radius $50 \mathrm{~cm}$, and inner height $100 \mathrm{~cm}$. On a spatial scale of meters or less, SPEs tend to assume an isotropic angular distribution, and this was reflected in the simulation for this case. Two shielding configurations were simulated: Configuration 1, with the cylindrical shell oriented such that the ends were parallel to the detector face, and Configuration 2, with the cylindrical shell oriented such that the ends were perpendicular to the detector face. In both configurations, the detector was located at the center of the cylindrical shell.

For Cases 1 and 2, separate simulations were performed for the August 1972 [39] and February 1956 [40] SPEs. These were historically-large events that are commonly used to investigate SPE dosimetry. The August 1972 SPE represents an event that is "soft", as it is comprised largely of lower-energy protons, while the February 1956 SPE represents an event that is "hard", as the fluence spectrum extends to higher proton kinetic energies. Instead of simulating the entire spectrum, each event was segmented into energy ranges consisting of protons with kinetic energies from $0.01 \mathrm{MeV}$ to $8.13 \mathrm{MeV}$; $8.13 \mathrm{MeV}$ to $65.5 \mathrm{MeV}$; 65.5 MeV to $100 \mathrm{MeV}$; and $100 \mathrm{MeV}$ to $2000 \mathrm{MeV}$. These ranges, determined using data from the National Institute of Standards and Technology StoppingPower and Range Tables for Protons database 32 for silicon, represent protons that will stop in the active volume, regardless of angle of incidence; protons that may stop in the active volume, depending on angle of incidence; protons that may slow in the active volume; and protons that will penetrate the active volume with little or no change in LET over most path lengths. 
Case 3 was devised to permit direct comparison of simulation results with data acquired at the Brookhaven National Laboratory (BNL) Tandem Van de Graaff Facility [41] for protons with kinetic energies of $5 \mathrm{MeV}$ and $20 \mathrm{MeV}$. The BNL Tandem Van de Graaff (TVdG) Facility has a rotation stage that is used to change angle of incidence of particles with respect to the detector face without needing to break vacuum. An image of HERA testing at the BNL TVdG Facility, with hardware mounted to the rotation stage, is shown in Figure1. Four angles with respect to normal incidence were tested: $0^{\circ}, 20^{\circ}, 40^{\circ}$, and $60^{\circ}$. Additional angles and proton kinetic energies were simulated to understand the behavior of protons that could be observed during an SPE.

\subsection{Timepix-based Detector Model}

A simplified model of the Timepix-based detector was constructed in PHITS. The active volume of the detector was defined as silicon with dimensions of $14.08 \mathrm{~mm}$ by $14.08 \mathrm{~mm}$ by $500 \mu \mathrm{m}$. A 256 by 256 array of bump bonds was modeled, each as a tin cube with side length $20 \mu \mathrm{m}$ centered on a pixel with side length $55 \mu \mathrm{m}$. Finally, the Timepix chip was modeled as a $700 \mu \mathrm{m}$ thick silicon element, with layers of nickel ( $2 \mu \mathrm{m}$ thick), gold ( $0.1 \mu \mathrm{m}$ thick), and copper (700 $\mu \mathrm{m}$ thick) beneath the silicon. The planar dimensions were $14.08 \mathrm{~mm}$ by $14.08 \mathrm{~mm}$ for the Timepix chip and all metallic layers.

\section{Results}

\subsection{Unshielded Detector}

Absorbed doses for the unshielded Timepix-based detector subjected to irradiation from a broad, mono-directional beam are shown in Figure 2 and Figure 3 for the August 1972 SPE and the February 1956 SPE, respectively ${ }^{3}$ As previously described, the spectra were split into four energy ranges to more clearly

\footnotetext{
${ }^{3}$ Uncertainties from Monte Carlo statistics are insignificant contributors to overall uncertainties for all simulated quantities. Therefore, in the present study, error bars are presented only for quantities that have other significant contributors to overall uncertainty.
} 
exhibit the impacts of slowing-down and stopped protons on the simulated absorbed doses.

\subsection{Shielded Detector}

Absorbed doses for the Timepix-based detector shielded by an aluminum cylindrical shell irradiated with an isotropic source are shown in Figure 4 and Figure 5, respectively. No simulations were performed for the $0.01 \mathrm{MeV}$ to 8.13 MeV energy range, since these protons do not have sufficient energy to penetrate the minimum aluminum shielding thickness.

\subsection{Benchmarking Mono-energetic Beam Simulations with Experiment}

Energy deposition data for $5 \mathrm{MeV}$ and $20 \mathrm{MeV}$ protons, which have ranges of $220 \mu \mathrm{m}$ and $2400 \mu \mathrm{m}$ in silicon, respectively [32, were acquired at the BNL TVdG Facility to perform advanced calibration 22] of Timepix-based radiation detectors to be used on the ISS. The advanced calibration process requires acquisition at multiple angles of incidence to obtain different values of energy deposition within the active volume. Example frames from data acquisition are shown in Figure 6 for $5 \mathrm{MeV}$ protons and Figure 7 for $20 \mathrm{MeV}$ protons. These frames show energy deposited per pixel, in units of kiloelectron-volts, as a function of spatial location on the Timepix. Each cluster of pixels results from energy deposition by a proton with the stated nominal kinetic energy. Acquisition of proton data at these energies presented an opportunity to further test the hypothesis by comparing simulations to experimental measurements.

First, absorbed dose response functions (Fig. 8) were calculated by simulation for the two energies at orientations corresponding to those used to acquire data. Next, simulated absolute absorbed doses were calculated by finding the product of the absorbed dose response function and estimated particle fluence. Unfortunately, the particle flux densities used in the data acquisition were too small to be measured using the facility detector, so the particle fluence was calculated using the proton cluster count as measured by the Timepix-based radiation detector and the projected area of the active volume with respect to 
the beam orientation. A comparison of measured and simulated absorbed doses is shown in Figure 9 for $5 \mathrm{MeV}$ protons and in Figure 10 for $20 \mathrm{MeV}$ protons. The subjective $95 \%$ confidence intervals for measured and simulated quantities were $\pm 5 \%$ and $\pm 10 \%$, respectively. The primary contributor to measurement error is conversion of time-over-threshold counts to energy deposition, while the primary contributor to simulation error is calculation of particle fluence.

\section{Discussion}

\subsection{Unshielded Detector}

The unshielded detector represents an extreme scenario resulting in the largest variation in absorbed dose as a function of angle of incidence with respect to the surface normal of the detector face. The proton kinetic energy ranges show consistent results in Figure 2 and Figure 3 . The $0.01 \mathrm{MeV}$ to $8.13 \mathrm{MeV}$ range exhibits the largest difference with angle. Ratio of absorbed dose for each angle to absorbed dose at normal incidence for this energy range is found to be equal to the ratio of projected areas for the angle and normal incidence. This result is expected, since (1) the energy deposition per particle is equal to the particle range for nearly all of the particles entering the volume, and (2) the number of particles entering the volume is proportional to the projected area of the detector with respect to the solid angle direction. A small increase in absorbed dose occurs at $45^{\circ}$, followed by a decrease at $90^{\circ}$ in the $8.13 \mathrm{MeV}$ to 65.5 MeV range, due to the off-setting effects of stopped particles and slowingdown particles (an increase in absorbed dose with increasing path length is expected for slowing-down particles because of the increase in $d E / d x$ associated with ranging). Small differences are observed for the two higher proton kinetic energy ranges. These results indicate that extremely large differences in absorbed dose can result from highly directional space radiation environments.

\subsection{Shielded Detector}

The shielded detector represents a more realistic exposure situation for a detector within a space vehicle during an SPE. Again, consistent results are ob- 
tained for the August 1972 SPE (Fig. 4) and February 1956 SPE (Fig. 5) for the two shielding configurations tested. The total event absorbed dose shows a difference of slightly less than $10 \%$, and is dominated by the $8.13 \mathrm{MeV}$ to $65.5 \mathrm{MeV}$ range, which exhibits a difference of slightly greater than $10 \%$. Configuration 1 results in a lower absorbed dose than Configuration 2 since the detector face is better shielded by the thicker cylindrical shell end caps in Configuration 1. In Configuration 2, the detector side is better shielded, but the absorbed dose from this direction is already attenuated, as shown for the unshielded detector. The overall difference between the two configurations is slightly less than $10 \%$ for both historical SPEs. If the detector were modeled as a point, the absorbed dose would be identical between the two configurations, since the environment is isotropic and the shielding distribution (ignoring solid angle dependence) is the same. This result highlights the importance of modeling the entire detector volume, especially for highly anisotropic active volumes.

\subsection{Benchmarking Mono-energetic Beam Simulations with Experiment}

In Figure 8, the $5 \mathrm{MeV}$ proton absorbed dose response function clearly exhibits a decrease with increasing angle from normal, resulting from the corresponding decrease in projected area presented to the beam by the detector. The $20 \mathrm{MeV}$ proton absorbed dose response function shows a slight increase with increasing angle from normal, as due to an increase in $d E / d x$ as the particles begin to range.

Comparison of absolute measured and simulated absorbed doses is excellent for $5 \mathrm{MeV}$ protons (Fig. 9), with percent differences in point estimates less than $4 \%$. Simulation and measurement for $20 \mathrm{MeV}$ protons agree to within $13 \%$, still acceptable considering the lack of an external beam monitor and associated uncertainties. An interesting result pertains to the ratio of simulated absorbed dose to measured absorbed dose. This ratio tends to decrease as angle increases, indicating that one or more physical processes are not adequately modeled, or there is some bias in the data analysis. It is likely that both are contributing to this trend. Recall that delta rays were not explicitly transported, effectively 
imposing an assumption of charged particle equilibrium for the detector. In vacuum, there should be a slight deficit of energy deposition, since some delta rays will escape the volume, and there is no matter near the detector volume to compensate. This effect would be maximized at normal incidence with the detector face, since this orientation presents the most area for potential delta ray escape, assuming the delta rays are forward directed, and would be more severe for higher-energy heavy charged particles, which generate higher energy delta rays; both trends are reflected in the results.

Additionally, energy cuts were used to define proton clusters in the analysis of measured data, since other clusters are associated with secondary electrons resulting from photon or primary electron interactions. Energy deposition cuts from stopped particles are straightforward to define, since the maximum energy deposition is equal to the particle kinetic energy. For slowing-down or penetrating particles, the definition is more difficult, since it requires defining a practical maximum for energy deposition by experience. Therefore, it is possible that a greater bias is present in calculation of particle fluence for $20 \mathrm{MeV}$ protons than for $5 \mathrm{MeV}$ protons, resulting in a larger percent difference and more prominent trend with increasing angle of incidence with the detector face. Future studies should explore the impacts of delta ray transport, particularly for detector measurements made in vacuum. Despite these sources of bias, the good agreement obtained between simulation and measurement indicate that the response functions shown in Figure 8 are reasonable, and reflect reality, at least to first order.

To further characterize detector response, proton kinetic energies from $1 \mathrm{MeV}$ to $1 \mathrm{GeV}$ were simulated for angles of incidence tested at the BNL TVdG Facility, and $90^{\circ}$. Absorbed dose response functions for these irradiation conditions are shown in Figure 11. For each angle, the response function increases with increasing proton kinetic energy to a maximum at the proton kinetic energy with range corresponding to nominal path length through the detector. The response function then decreases with increasing proton kinetic energy, as expected from the Bethe-Bloch formulation. Ratios of response functions at various angles to 
the response function at normal incidence were also computed (Fig. 12). The stopped, slowing-down, and penetrating energy regimes are more clearly exhibited. For proton kinetic energies below about $8 \mathrm{MeV}$, the response function ratio is equal to the ratio of projected areas presented by the detector to the beam. Above $8 \mathrm{MeV}$, protons are still in the process of slowing down for larger angles. The ratio decreases onces the protons begin to penetrate the detector, but are still in the process of slowing down. Finally, the ratio of response functions converges to unity as the protons penetrate the detector with little change in $d E / d x$ over the path lengths through the detector.

\section{Conclusion}

Slowing-down and stopped charged particles were hypothesized to cause angular dependence for absorbed dose measurements in space radiation environments. To test the hypothesis, simulations were performed with the detector exposed to SPE environments under varying shielding conditions and solid angle distributions. Additionally, mono-energetic proton measurements were compared with simulation, and good agreement was obtained. The results indicate that the hypothesis is correct: absorbed dose differences with detector orientation are attributed to slowing-down and stopped charged particles.

Large variations in magnitude of the effect were observed, depending on shielding and the degree of field anisotropy. Worst-case differences are found for the unshielded detector exposed to a mono-directional proton fluence. Even under an isotropic boundary condition, absorbed dose differences exist, if the shielding is anisotropic. The results of this study indicate that detector volume should be modeled along with the solid angle distribution of the space radiation environment and shielding. The orientation of the detector with respect to an absolute coordinate system is important for interpreting absorbed dose measurements.

Although dosimetric quantities such as absorbed dose and dose equivalent continue to serve a role in space radiation protection, the ultimate goal is to 
characterize the flux density spectrum as a function of particle type and kinetic energy through probabilistic particle classification. This will allow radiation experts to unfold the external environment, transport the external environment through all relevant shielding (including vehicle, supplies, and body self-shielding), and calculate organ-specific risks. Detector volumetric and orientation effects will still impact this new space radiation environment monitoring paradigm, and must be characterized to ensure the most accurate astronaut radiation risk analyses.

\section{Acknowledgements}

This work was supported by the Kansas State University Department of Mechanical and Nuclear Engineering and Office of Engineering Research and Graduate Programs. It was also supported by the NASA Advanced Exploration Systems RadWorks project through Bioastronautics Contract NAS9-02078 and Human Health and Performance Contract NNJ15HK11B.

\section{References}

[1] R. Mewaldt, Solar energetic particle composition, energy spectra, and space weather, Space Science Reviews 124 (1) (2006) 303-316.

[2] J. A. Simpson, Elemental and isotopic composition of the galactic cosmic rays, Annual Review of Nuclear and Particle Science 33 (1) (1983) 323-382.

[3] J. A. Van Allen, The geomagnetically trapped corpuscular radiation, Journal of Geophysical Research 64 (11) (1959) 1683-1689.

[4] E. Codman, A study of the cases of accidental X-ray burns hitherto recorded, The Philadelphia Medical Journal 9 (10) (1902) 438-442,499503.

[5] F. A. Cucinotta, M. Durante, Cancer risk from exposure to galactic cosmic rays: implications for space exploration by human beings, The lancet oncology 7 (5) (2006) 431-435. 
[6] F. A. Cucinotta, M. Alp, F. M. Sulzman, M. Wang, Space radiation risks to the central nervous system, Life Sciences in Space Research 2 (2014) 54 $-69$.

[7] V. A. Convertino, Status of cardiovascular issues related to space flight: Implications for future research directions, Respiratory Physiology \& Neurobiology 169 (2009) S34-S37.

[8] J. E. Baker, J. E. Moulder, J. W. Hopewell, Radiation as a risk factor for cardiovascular disease, Antioxidants \& redox signaling 15 (7) (2011) 1945-1956.

[9] F. Cucinotta, F. Manuel, J. Jones, G. Iszard, J. Murrey, B. Djojonegro, M. Wear, Space radiation and cataracts in astronauts, Radiation Research 156 (5) (2001) 460-466.

[10] L. T. Chylack Jr, L. E. Peterson, A. H. Feiveson, M. L. Wear, F. K. Manuel, W. H. Tung, D. S. Hardy, L. J. Marak, F. A. Cucinotta, NASA study of cataract in astronauts (NASCA). report 1: Cross-sectional study of the relationship of exposure to space radiation and risk of lens opacity, Radiation Research 172 (1) (2009) 10-20.

[11] S. Hu, M.-H. Y. Kim, G. E. McClellan, F. A. Cucinotta, Modeling the acute health effects of astronauts from exposure to large solar particle events, Health Physics 96 (4) (2009) 465-476.

[12] J. P. Kahn, C. T. Liverman, M. A. McCoy (Eds.), Health standards for long duration and exploration spaceflight: ethics principles, responsibilities, and decision framework, National Academies Press, 2014.

[13] NASA Space Flight Human-System Standard Volume 1, Revision A: Crew Health, NASA-STD-3001, National Aeronautics and Space Administration, Washington, DC (2015).

[14] M. Shavers, N. Zapp, R. Barber, J. Wilson, G. Qualls, L. Toupes, S. Ramsey, V. Vinci, G. Smith, F. Cucinotta, Implementation of ALARA radiation 
protection on the ISS through polyethylene shielding augmentation of the Service Module Crew Quarters, Advances in Space Research 34 (6) (2004) 1333 - 1337, space Life Sciences: Radiation Risk Assessment and Radiation Measurements in Low Earth Orbit.

[15] D. Zhou, E. Semones, R. Gaza, S. Johnson, N. Zapp, K. Lee, T. George, Radiation measured during ISS-Expedition 13 with different dosimeters, Advances in Space Research 43 (8) (2009) 1212-1219.

[16] D. Perez-Nunez, L. Braby, Replacement tissue-equivalent proportional counter for the International Space Station, Radiation Protection Dosimetry $143(2-4)(2010)$ 394-397.

[17] D. Hassler, C. Zeitlin, R. Wimmer-Schweingruber, S. Böttcher, C. Martin, J. Andrews, E. Böhm, D. Brinza, M. Bullock, S. Burmeister, et al., The Radiation Assessment Detector (RAD) investigation, Space Science Reviews 170 (1-4) (2012) 503-558.

[18] K. Lee, J. Flanders, E. Semones, T. Shelfer, F. Riman, Simultaneous observation of the radiation environment inside and outside the ISS, Advances in Space Research 40 (11) (2007) 1558-1561.

[19] X. Llopart, R. Ballabriga, M. Campbell, L. Tlustos, W. Wong, Timepix, a 65k programmable pixel readout chip for arrival time, energy and/or photon counting measurements, Nuclear Instruments and Methods in Physics Research Section A: Accelerators, Spectrometers, Detectors and Associated Equipment 581 (1) (2007) 485-494.

[20] M. Campbell, 10 years of the Medipix2 Collaboration, Nuclear Instruments and Methods in Physics Research Section A: Accelerators, Spectrometers, Detectors and Associated Equipment 633 (2011) S1 - S10, 11th International Workshop on Radiation Imaging Detectors (IWORID).

[21] J. Jakubek, Precise energy calibration of pixel detector working in timeover-threshold mode, Nuclear Instruments and Methods in Physics Re- 
search Section A: Accelerators, Spectrometers, Detectors and Associated Equipment 633 (2011) S262-S266.

[22] M. Kroupa, T. Campbell-Ricketts, A. Bahadori, A. Empl, Techniques for precise energy calibration of particle pixel detectors, Review of Scientific Instruments 88 (3) (2017) 033301.

[23] N. Stoffle, L. Pinsky, M. Kroupa, S. Hoang, J. Idarraga, C. Amberboy, R. Rios, J. Hauss, J. Keller, A. Bahadori, E. Semones, D. Turecek, J. Jakubek, Z. Vykydal, S. Pospisil, Timepix-based radiation environment monitor measurements aboard the International Space Station, Nuclear Instruments and Methods in Physics Research Section A: Accelerators, Spectrometers, Detectors and Associated Equipment 782 (2015) 143-148.

[24] M. Kroupa, A. Bahadori, T. Campbell-Ricketts, A. Empl, S. M. Hoang, J. Idarraga-Munoz, R. Rios, E. Semones, N. Stoffle, L. Tlustos, et al., A semiconductor radiation imaging pixel detector for space radiation dosimetry, Life Sciences in Space Research 6 (2015) 69-78.

[25] A. A. Bahadori, E. J. Semones, R. Gaza, M. Kroupa, R. R. Rios, N. N. Stoffle, T. Campbell-Ricketts, L. S. Pinsky, D. Turecek, Battery-operated Independent Radiation Detector Data Report from Exploration Flight Test 1 NASA/TP-2015-218575, NASA Johnson Space Center, Houston, TX (2015).

[26] F. Badavi, X. Xu, T. Slaba, M. Clowdsley, A model for the Low Earth Orbit secondary proton environment based on the Alpha Magnetic Spectrometer 1 (AMS-1) measurements, 2017 NASA Human Research Program Investigators' Workshop, January 23-26, 2017.

[27] A. S. Johnson, Personal Communication, July 25, 2016.

[28] T. Berger, S. Burmeister, D. Matthiä, B. Przybyla, G. Reitz, P. Bilski, M. Hajek, L. Sihver, J. Szabo, I. Ambrozova, F. Vanhavere, R. Gaza, E. Semones, E. G. Yukihara, E. R. Benton, Y. Uchihori, S. Kodaira, H. Kita- 
mura, M. Boehme, DOSIS \& DOSIS 3D: radiation measurements with the DOSTEL instruments onboard the Columbus Laboratory of the ISS in the years 2009-2016, Journal of Space Weather and Space Climate 7 (2017) A8.

[29] S. Drobyshev, V. Benghin, Estimation of the effect of orientation of International Space Station on the dose rate in Stations Service Module when passing through the South-Atlantic anomaly region, Cosmic Research 49 (5) (2011) 399-406.

[30] N. Stoffle, L. Pinsky, Identification of stopping ions in a silicon Timepix detector, Nuclear Instruments and Methods in Physics Research Section A: Accelerators, Spectrometers, Detectors and Associated Equipment, Submitted.

[31] D. S. McGregor, J. K. Shultis, Reporting detection efficiency for semiconductor neutron detectors: A need for a standard, Nuclear Instruments and Methods in Physics Research Section A: Accelerators, Spectrometers, Detectors and Associated Equipment 632 (1) (2011) 167 - 174.

[32] M. Berger, J. Coursey, M. Zucker, J. Chang, ESTAR, PSTAR, and ASTAR: Computer Programs for Calculating Stopping-Power and Range Tables for Electrons, Protons, and Helium Ions (Version 1.2.3)[Online], National Institute of Standards and Technology, Gaithersburg, MD (2005).

URL http://physics.nist.gov/Star

[33] J. C. Pickel, J. T. Blandford, Cosmic-ray-induced errors in MOS devices, IEEE Transactions on Nuclear Science 27 (2) (1980) 1006-1015.

[34] T. C. Slaba, S. R. Blattnig, F. F. Badavi, Faster and more accurate transport procedures for HZETRN, Journal of Computational Physics 229 (24) (2010) 9397-9417.

[35] J. W. Wilson, T. C. Slaba, F. F. Badavi, B. D. Reddell, A. A. Bahadori, Ad- 
vances in NASA radiation transport research: 3DHZETRN, Life Sciences in Space Research 2 (2014) 6-22.

[36] T. Sato, K. Niita, N. Matsuda, S. Hashimoto, Y. Iwamoto, S. Noda, T. Ogawa, H. Iwase, H. Nakashima, T. Fukahori, et al., Particle and heavy ion transport code system, PHITS, version 2.52, Journal of Nuclear Science and Technology 50 (9) (2013) 913-923.

[37] A. Boudard, J. Cugnon, J.-C. David, S. Leray, D. Mancusi, New potentialities of the Liege intranuclear cascade model for reactions induced by nucleons and light charged particles, Physical Review C 87 (1) (2013) 014606.

[38] H. Hirayama, Y. Namito, W. R. Nelson, A. F. Bielajew, S. J. Wilderman, The EGS5 code system SLAC-R-730/KEK-2005-8, United States. Department of Energy (2005).

[39] J. H. King, Solar proton fluences for 1977-1983 space missions, Journal of Spacecraft and Rockets 11 (6) (1974) 401-408.

[40] P. Freier, W. Webber, Exponential rigidity spectrums for solar-flare cosmic rays, Journal of Geophysical Research 68 (6) (1963) 1605-1629.

[41] P. Thieberger, D. Barton, J. Benjamin, C. Chasman, H. Foelsche, H. Wegner, Tandem injected relativistic heavy ion facility at Brookhaven, present and future, Nuclear Instruments and Methods in Physics Research Section A: Accelerators, Spectrometers, Detectors and Associated Equipment $268(2-3)(1988)$ 513-521. 


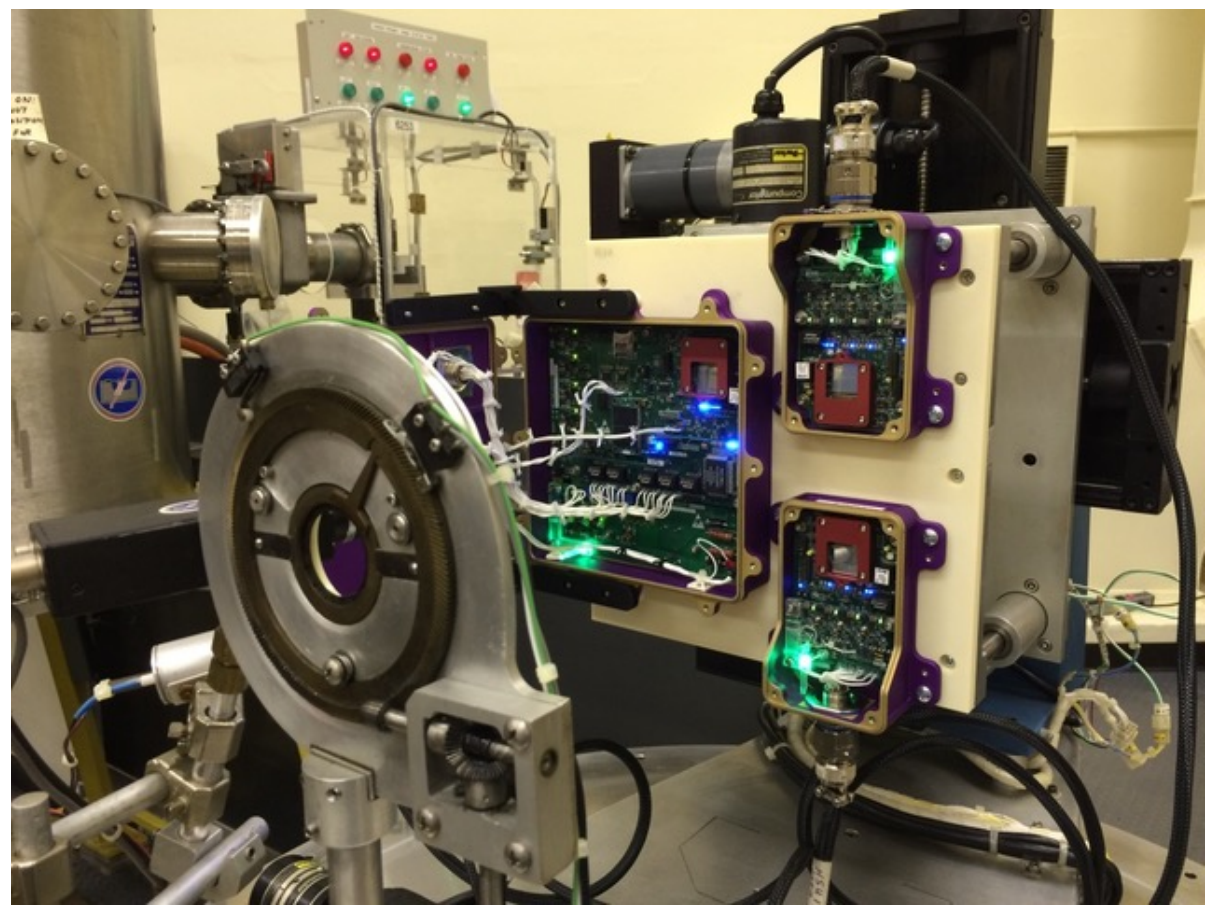

Figure 1: HERA test set-up at BNL TVdG Facility 


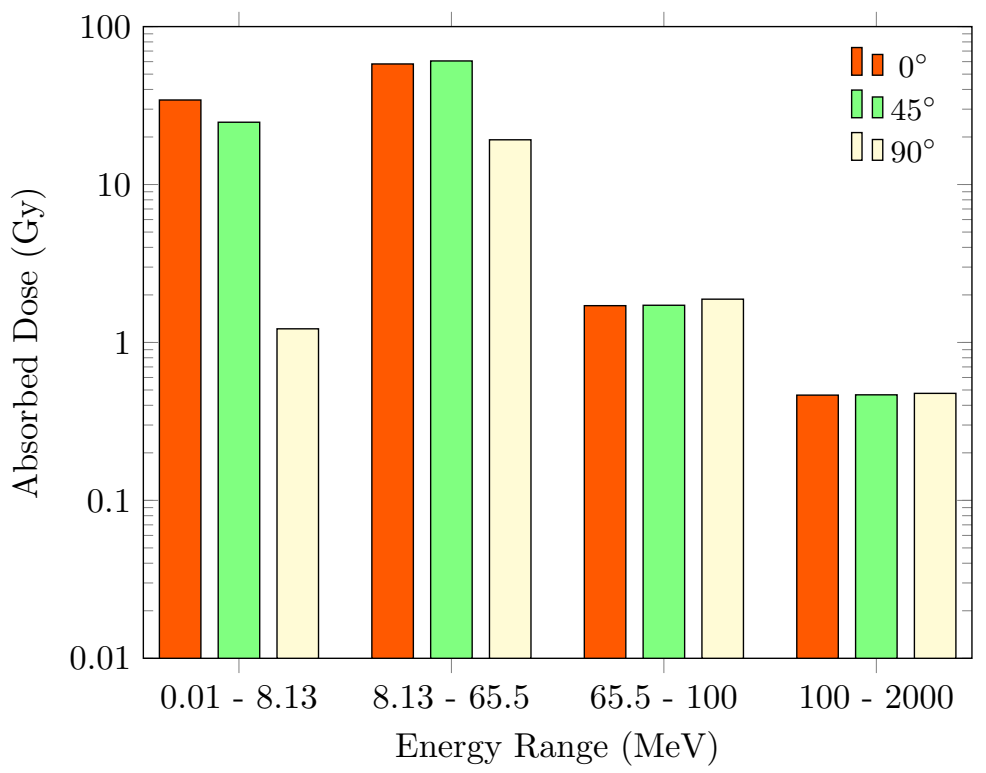

Figure 2: Absorbed dose for unshielded detector exposed to mono-directional August 1972 SPE 


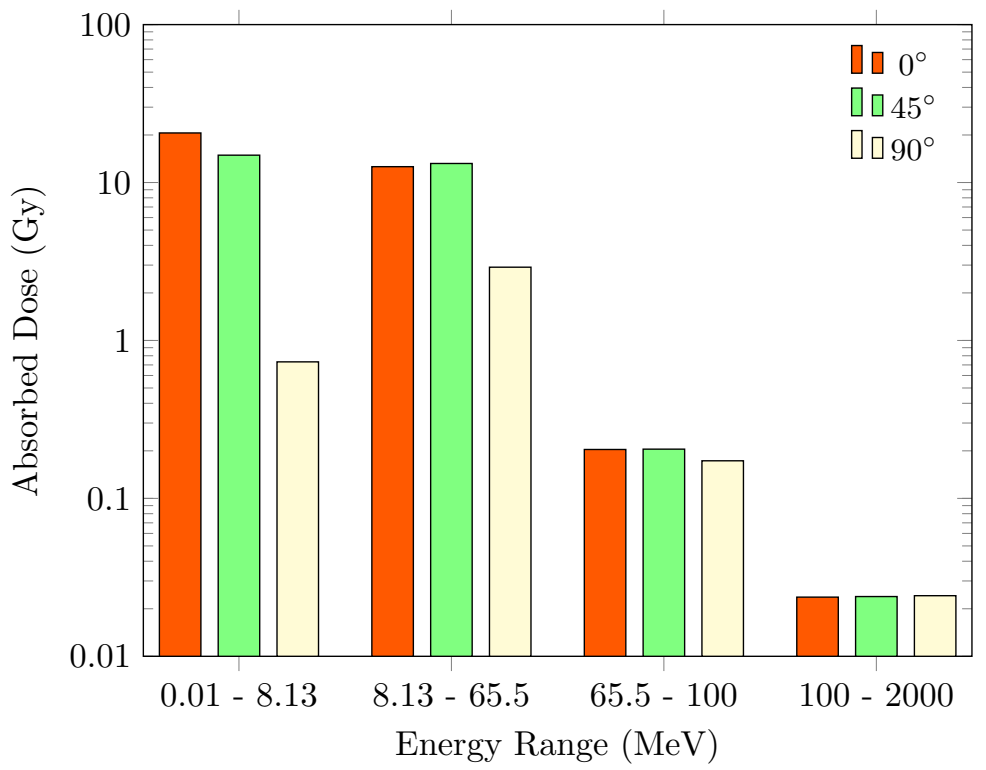

Figure 3: Absorbed dose for unshielded detector exposed to mono-directional February 1956 SPE 


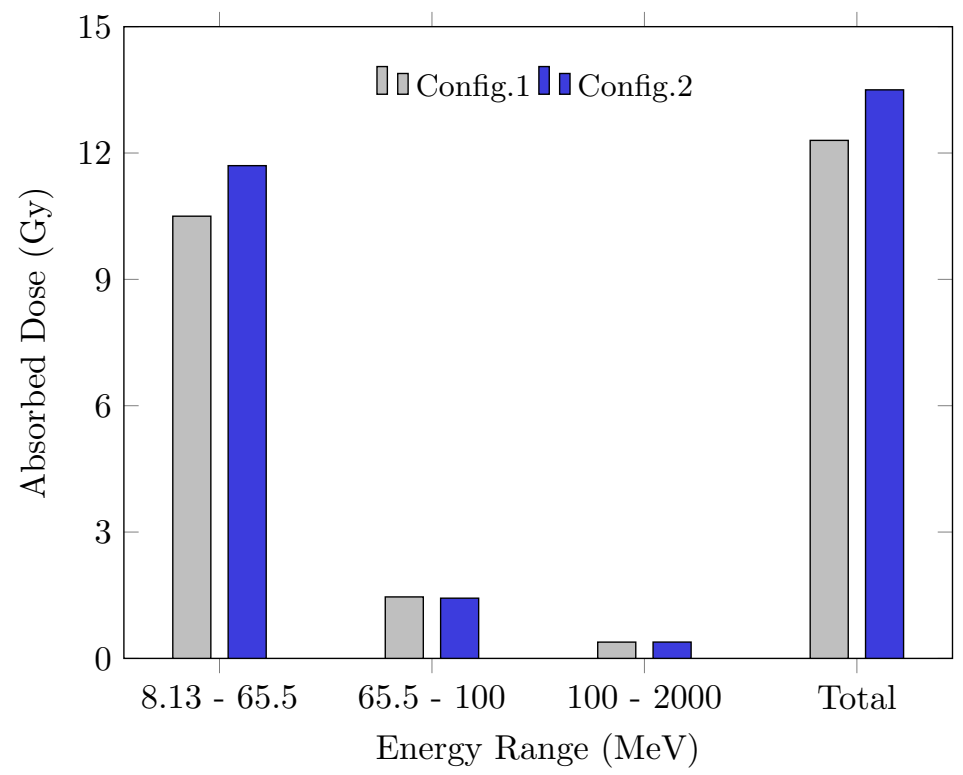

Figure 4: Absorbed dose for shielded detector exposed to isotropic August 1972 SPE 


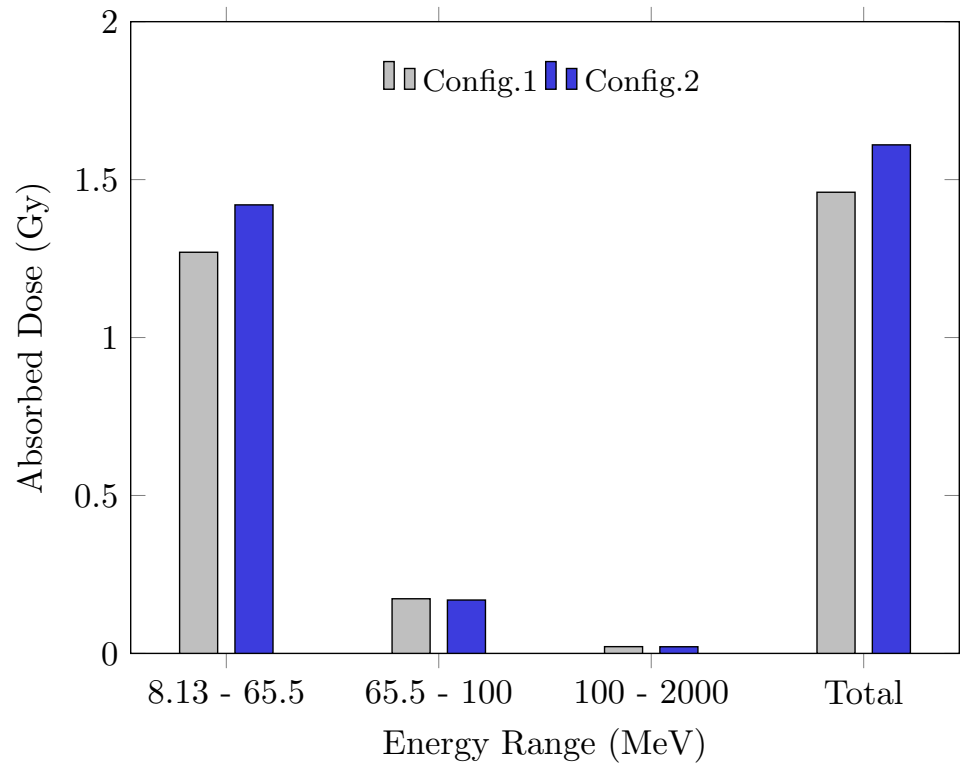

Figure 5: Absorbed dose for shielded detector exposed to isotropic February 1956 SPE 


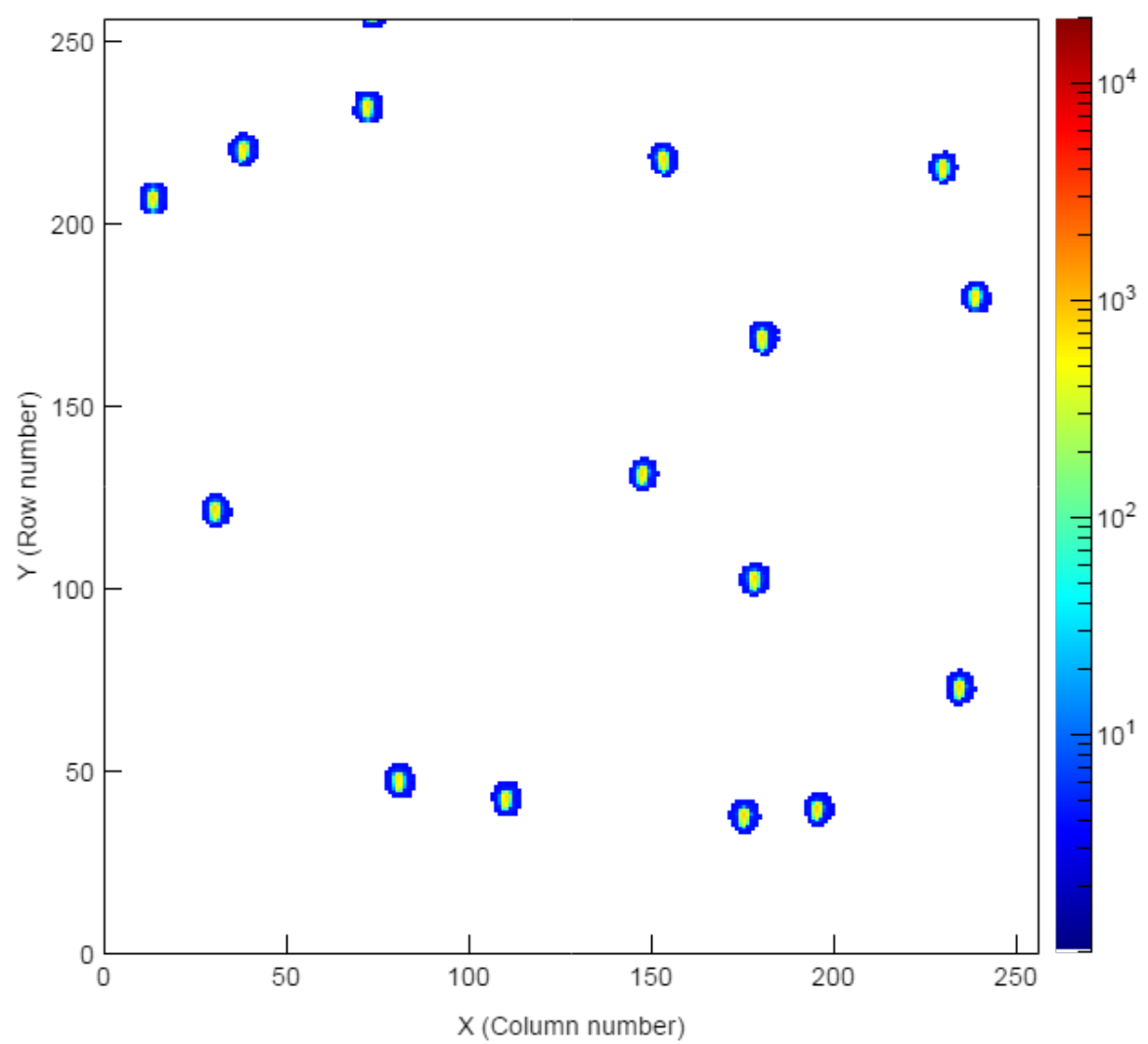

Figure 6: Example frame for $5 \mathrm{MeV}$ protons acquired at 60 degrees from normal incidence 


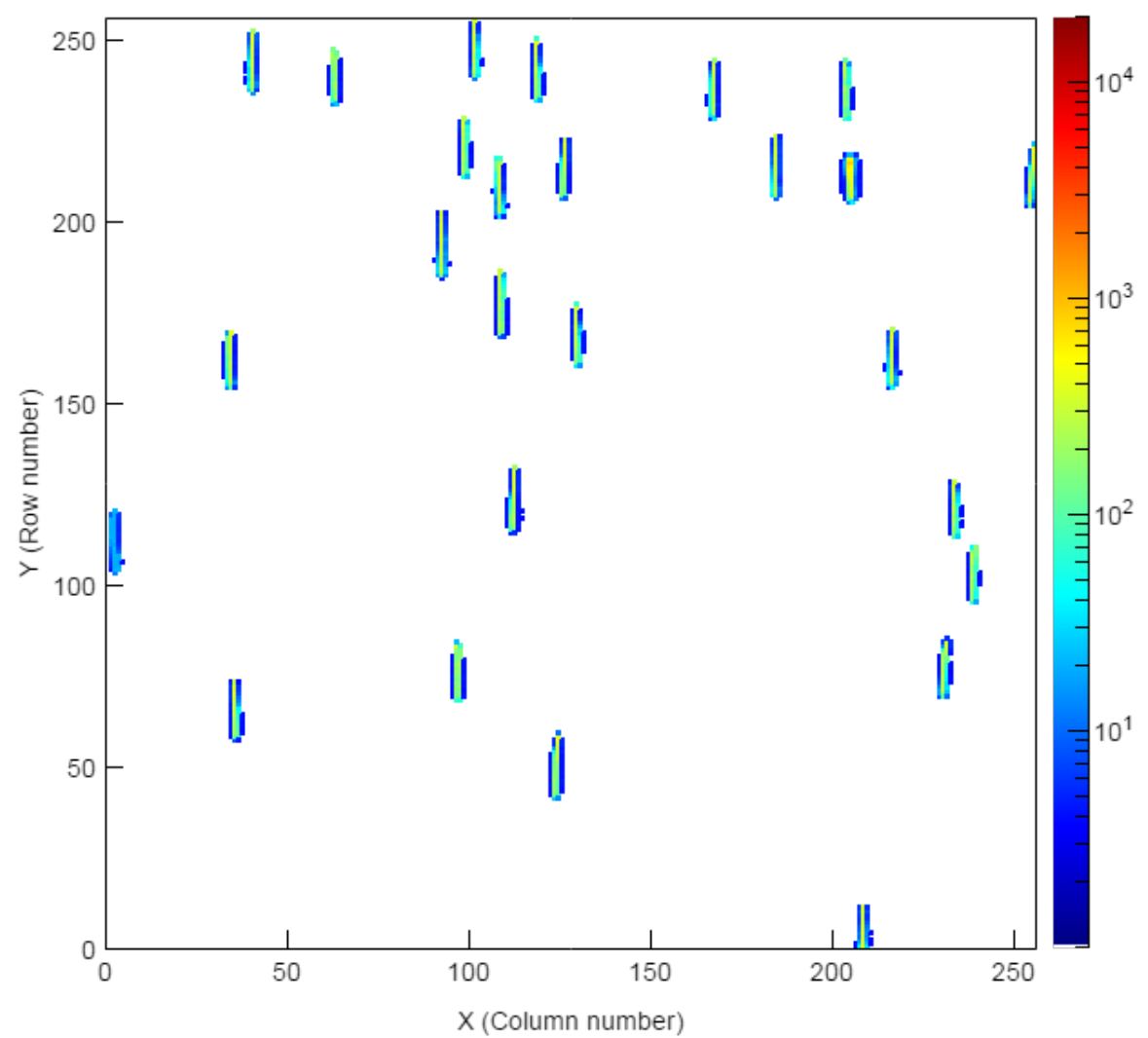

Figure 7: Example frame for $20 \mathrm{MeV}$ protons acquired at 60 degrees from normal incidence 


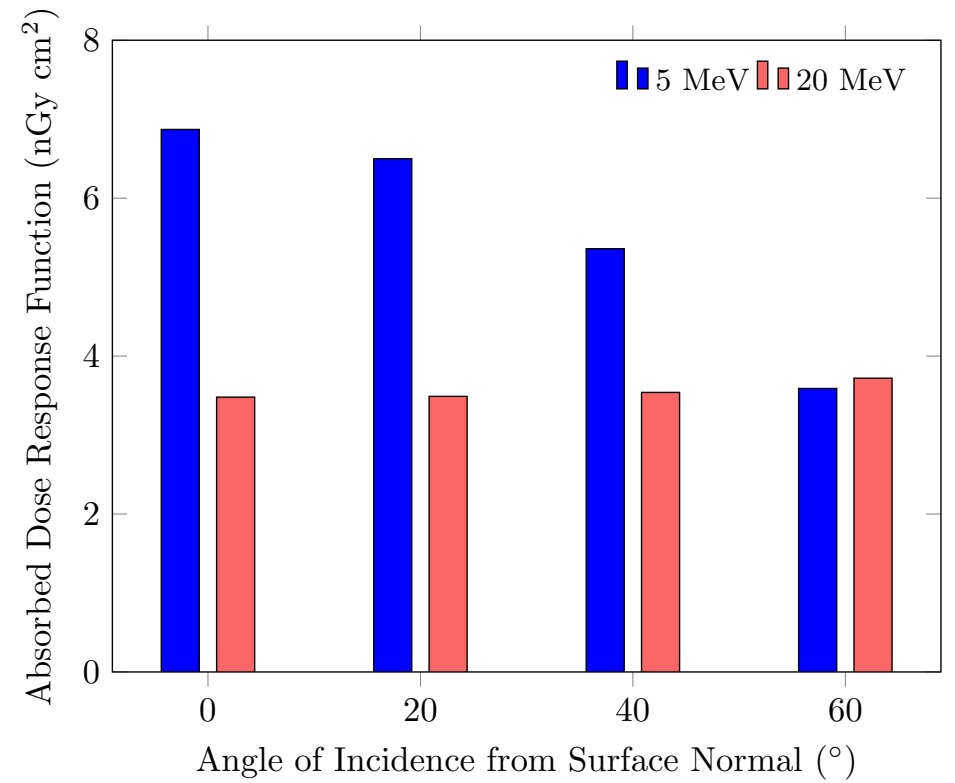

Figure 8: Simulated absorbed dose response functions for detector exposed to mono-directional protons 


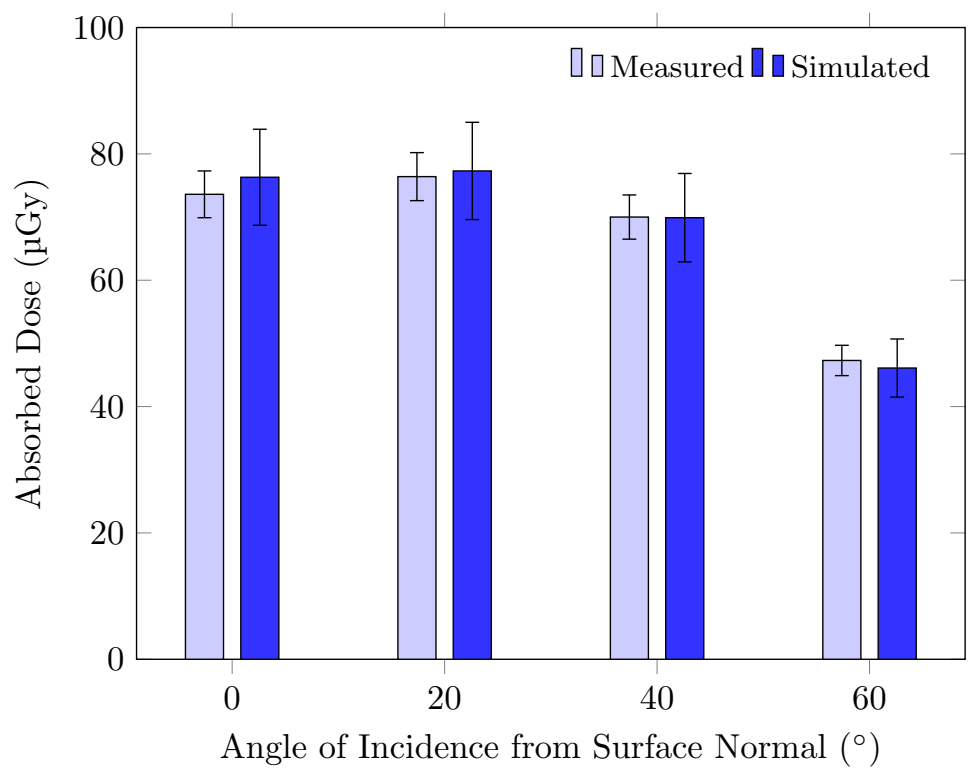

Figure 9: Absorbed dose comparison for mono-directional $5 \mathrm{MeV}$ protons 


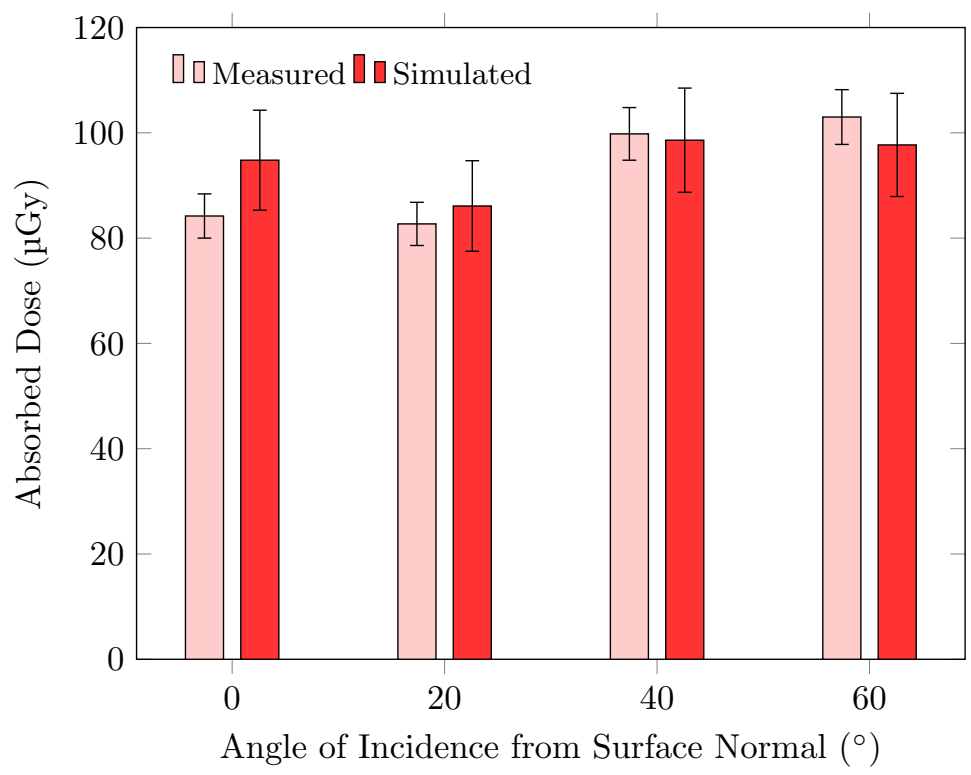

Figure 10: Absorbed dose comparison for mono-directional $20 \mathrm{MeV}$ protons 


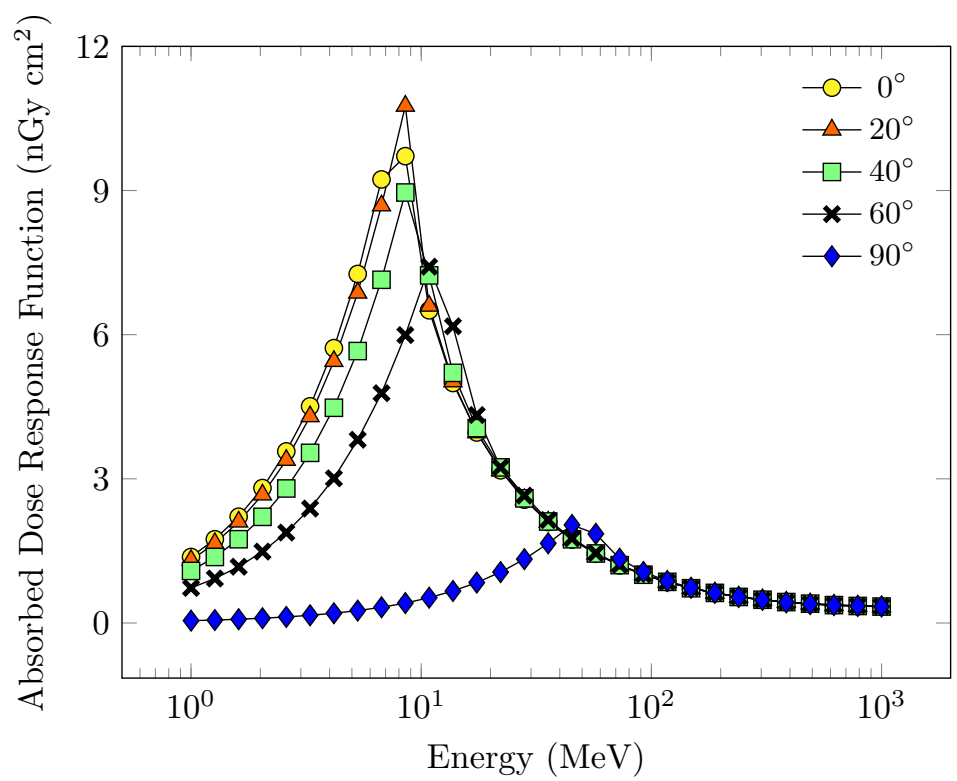

Figure 11: Absorbed dose response functions for mono-directional beams at various angles of incidence 


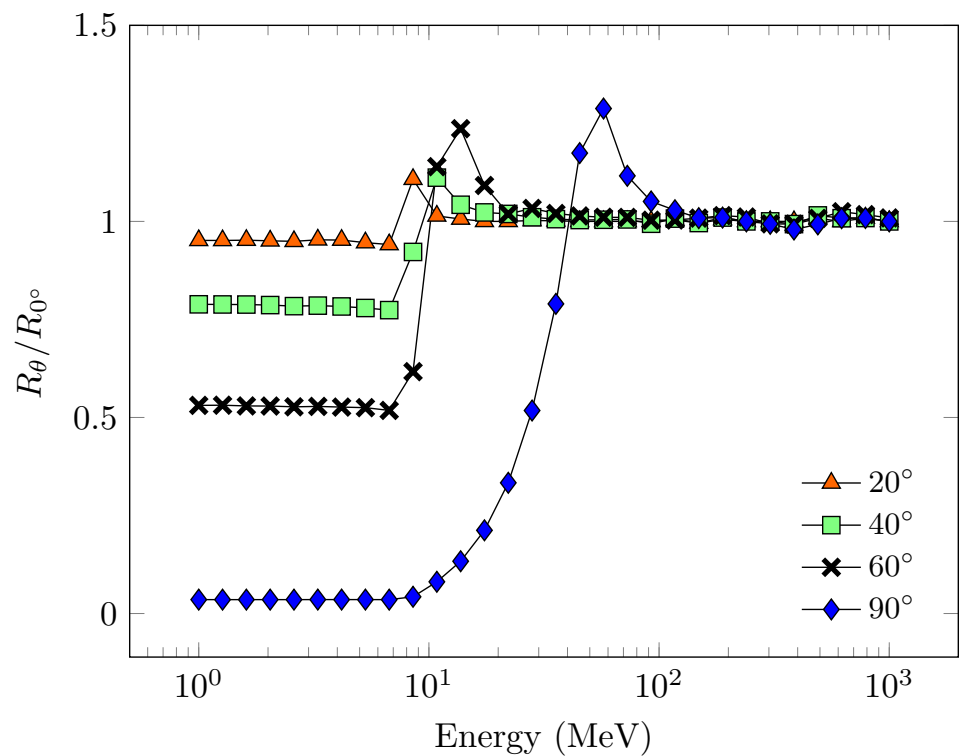

Figure 12: Ratio of absorbed dose response functions for mono-directional beams at various angles of incidence with respect to absorbed dose response function at normal incidence

(C) 2018. This manuscript version is made available under the CCBY-NC-ND 4.0 license http://creativecommons.org/licenses/bync-nd/4.0/ 